\title{
A new barrier at mucosal surfaces
}

\section{C6 \\ the phage had \\ a protective effect on the epithelium.}

For many pathogens, the initial point of entry into the host is the mucosal epithelium, and the host immune system deploys several defence mechanisms at this surface, including the mucus itself. Now, Barr et al. show that phages might constitute an additional antibacterial defence mechanism at host mucosal surfaces.

The authors sampled mucosal surfaces from a range of organisms and found that the phage-to-bacterium ratio was about four-fold higher at these surfaces than in neighbouring environments. Testing phage $\mathrm{T} 4$ adherence to human cell lines showed that more phages adhered to mucus-producing cells than to non-mucus-producing cells and that chemical removal of the mucus reduced phage adherence.

Many phages in the human intestine encode proteins with hypervariable immunoglobulin (Ig)-like domains, which are involved in cell adhesion in many organisms. The T4 surface protein Hoc (highly antigenic outer capsid) contains four such domains, which the authors speculated might bind mucus components. Indeed, the increase in the number of T4 phages adhering to mucin-coated agar compared with non-coated agar was considerably greater for wildtype phages than for a Hoc-deficient mutant. Moreover, the authors found that although wild-type T4 could bind diverse mammalian glycans, it had a particular affinity for those commonly found in mucin glycoproteins, whereas the Hoc-deficient phage displayed limited binding to all 610 glycans tested. Thus, phage T4 seems to adhere to mucus through the interaction of $\mathrm{Hoc}$ with mucin glycans.

Lytic phages such as T4 kill bacterial strains that compete with their host strain. To test whether such lytic activity could reduce bacterial colonization at mucosal surfaces, the authors assessed the effects of Escherichia coli exposure on mucusproducing tissue culture cells that

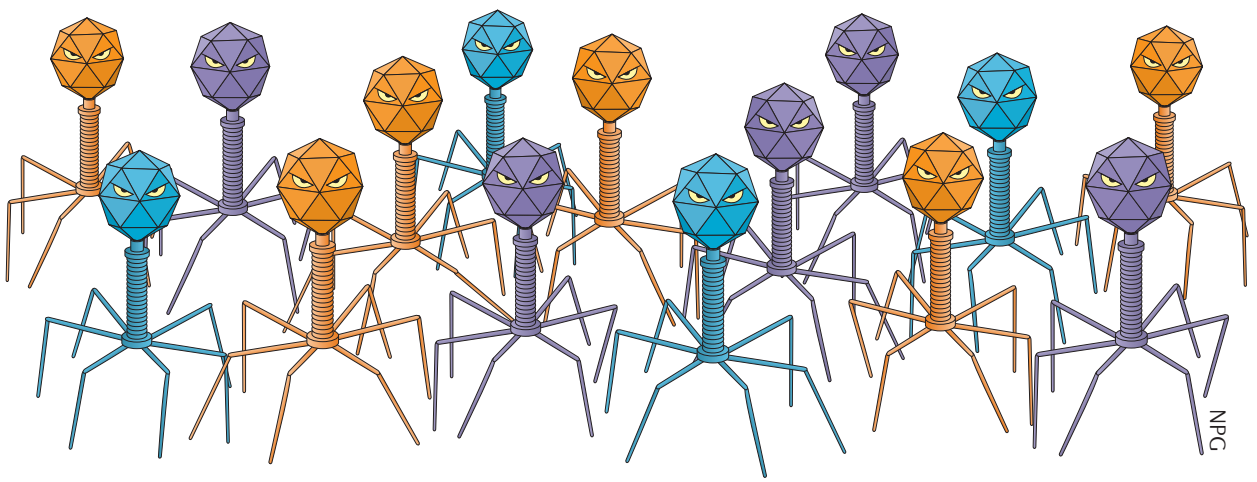

had been pre-treated with phage T4. Both bacterial attachment and epithelial cell death were significantly lower for T4-treated cells than for non-treated cells, indicating that the phage had a protective effect on the epithelium.

On the basis of these findings, Barr et al. present a model in which lytic phages bind glycan components of mucin through the Ig-like domains of surface proteins, forming an antimicrobial layer that decreases mucus colonization by bacteria and thus protects the underlying epithelial cells from infection. They further propose that the hypervariability of the Ig-like domains and the dynamic nature of the mucus, which is not only variable in structure but also continually sloughed off at the outer surface, would allow rapid phage adaptation to both changes in the mucus and bacterial invasion.

The relevance of this model in the gut environment and whether it applies to temperate phages (which are common in the gut) remain to be seen, and the potential to therapeutically exploit such phage-mediated immunity also needs to be explored. This work will undoubtedly be a spring-board to many key discoveries in the future.

Lucie Wootton 